\title{
Acoustic correlates of the French Accentual Phrase in Lifou (New Caledonia)
}

\author{
Catalina Torres $^{1,2}$, Janet Fletcher ${ }^{1,2}$, Gillian Wigglesworth ${ }^{1,2}$ \\ ${ }^{1}$ University of Melbourne, Australia; ${ }^{2}$ ARC Centre of Excellence for the Dynamics of Language, \\ Australia \\ catalinatestudent.unimelb.edu.au, j.fletchereunimelb.edu.au
}

\begin{abstract}
This paper investigates the realization of the Accentual Phrase (AP) in Lifou French by bilingual speakers of Drehu and French. In French prominence is marked within a phrasal domain and the AP represents the lowest tonally marked prosodic constituent. Although still controversial, increasingly, there have been contributions arguing for a further prosodic level, the intermediate phrase (ip) between the AP and the Intonation Phrase (IP). In this study, it is shown that Lifou French uses the same tonal patterns as found for Standard French. Additionally, further evidence for the existence of another prosodic level after the AP is found. However, while in Standard French an increased F0 rise and final vowel lengthening have been shown to mark the ip-boundary, an expanded pitch span represents the more salient cue to mark this in Lifou French.
\end{abstract}

Index Terms: Accentual Phrase, Bilingual French, intermediate phrase, Prosodic Hierarchy

\section{Introduction}

New Caledonia is sui generis ${ }^{1}$ a collectivity of France situated in the South Pacific about $17000 \mathrm{~km}$ away from mainland France. Lifou is an island counting no more than 9000 inhabitants whose indigenous language is Drehu, an Oceanic language [1]. From the 1950s the French established in New Caledonia a mass education system aligned to the metropolitan French syllabus [2] and today there are almost no monolingual Drehu speakers on Lifou. The phonetics and phonology of French have been studied much more extensively than those of Drehu. While French has been analysed as a language with phrasal prominence marking [3] Drehu has been impressionistically described as having lexical prominence marking $[4,5]$. Previous studies on French contact varieties $[6,7]$ suggest that when in contact with a language with different prominence marking, the speech flow in French would be segmented into smaller chunks, comparable to word prosodic segmentation. However, to our knowledge there are no detailed phonetic studies of bilingual speakers of French living in remote areas such as Lifou. In this study, laboratory phonology methods are used in order to study bilingual speech production. More precisely, we investigate the realization of the French Accentual Phrase and its acoustic correlates in the speech production of five Drehu French bilingual speakers. The Accentual Phrase (AP) represents the lowest tonally marked prosodic constituent in French [3]. Although the position of stress is fixed at the word level, its realisation relies upon the location of a word within a phrase. The metrical patterns of the AP are linked to the presence of two distinct stress types which are assigned at the phrasal level: an obligatory phrasefinal, primary stress, and an optional phrase-initial, secondary

${ }^{1}$ After the Noumea accord from 1998 New Caledonia became "une collectivité d'outre-mer à statut particulier" hence a collectivity with a special status different to its old one as overseas territory. stress. The AP consists of one or more content words (Wc), optionally preceded by one or more function words (Wf). Factors such as word length, syntactic and semantic structure as well as speech rate of individual speakers can have an influence on AP length in terms of number of syllables [8], although its maximal length has been estimated at 7 syllables [9]. Jun and Fougeron [3] propose an autosegmental-metrical analysis of the AP and the notation /LHiLH*/ as the underlying tonal pattern. The Hi represents the optional phrase-initial prominence, and $\mathrm{H}^{*}$ the phrase-final prominence, the latter described as being stronger in pitch and duration. According to Welby [8], the initial LHi sequence represents an edge tone which is structurally different from the final $\mathrm{LH}^{*}$ rise which is a pitch accent. The initial rise is associated with the constituent boundary, which represents the left edge of the AP and is not linked to any particular syllable within it. The final rise $\left(\mathrm{LH}^{*}\right)$, marks the right boundary of an AP. It has a double association, then, and $\mathrm{LH}^{*}$ marks the right edge of an AP, but $\mathrm{H}^{*}$ is at the same time associated with the stressed syllable of an AP. The $\mathrm{LH}^{*}$ tone is considered a pitch accent because part of the tone is associated with a stressed syllable at the phrasal level. However, note that [3] and [8] agree on the definition of pitch accent for French as being different from that used for Germanic languages where it is associated with a lexically stressed syllable. Finally, the realisation of the $\mathrm{L}$ tone is said to be more variable due to its lack of association with any specific syllable. Its realisation can be on the penultimate syllable of the AP, meaning on the syllable immediately preceding the $\mathrm{H}^{*}$, but it is sometimes realised on the final syllable together with $\mathrm{H}^{*}$. As exemplified in Figure 1, $[10,11]$ have pointed out that tonal targets within the AP can be undershot, and apart from the canonical pattern, five other tonal patterns are identified for French.

The prosodic hierarchy in French and the intermediate phrase (ip) as a next level above the AP are not uncontroversial. In [10] the authors argue for an intermediate phrase level between the AP and IP (intonational phrase), but this analysis is not maintained later [3] and also [12] argues against it. However, more recent studies $[13,14,15]$ devoted to the internal structure of the intonation phrase in French find evidence for the intermediate phrase level. According to [15], phonological and phonetic cues which are stronger than those found for the AP boundary signal the ip boundary. More precisely, the authors find that within the ip final syllables are produced with lower F0 values when in non-final position (declination) but register shift and a complete reset of F0 is found when immediately preceding an ip right edge. Additionally, they find greater vowel lengthening within ip-final syllables than within non-final ones.

\section{Research questions}

This pilot study was conducted in order to investigate the realization of the AP in read speech, in Lifou French. For this 


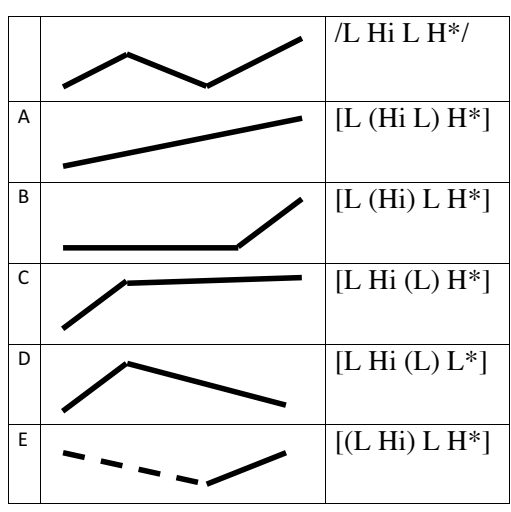

Figure 1: Canonical and five types of surface realizations of AP $\left(/ \mathrm{LHiLH} H^{*} /\right)$ when not all four underlying tones are realized. The tone $(s)$ in a parentheses refers to the tone $(s)$ not realized due to undershoot. In pattern $(E)$ the dotted line indicates the fall from the $H^{*}$ of a preceding AP.

purpose we based our study on a previous experiment that analysed the AP in Standard French [11]. Our first aim was to establish the tonal patterns used (shown in Figure 1) and find their distribution within our experimental data. We were also interested in verifying whether the realization of the two tonal targets $\mathrm{Hi}$ and $\mathrm{H}^{*}$ correspond to that found for Standard French. Second, we noted the insertion of pauses following the target AP and hypothesised the insertion of a further prosodic level, an intermediate phrase (ip). According to [15], the boundary tone of an ip in sentence medial position is marked through register shift and complete reset. Conversely, a sequence of APs in an utterance would show declination after every AP. To test this hypothesis we assumed that the boundary tone of the ip should show differences in fundamental frequency (higher F0) and also in duration (longer) of the final syllable when compared to APs where no break was included. Third, we further hypothesised that in order to mark the ip boundary, apart from an increase in fundamental frequency at the boundary tone target, a more extended pitch span could be employed. This would be realised through the expansion of the final rise with a lowered $\mathrm{L}$ and a raised $\mathrm{H}^{*}$ tone.

\section{Materials and Method}

\subsection{Participants}

Five female speakers from Lifou (age 29 - 47) were recorded in Lifou. Participants responded to a linguistic questionnaire similar to the Bilingual Language Profile [16]. All had acquired French and Drehu during childhood (starting at no later than 7 years with either language), were schooled in French and had varying degrees of school instruction in Drehu (0 to 10 years). Participants were not only literate in French but also in Drehu. Additionally, they work in the local community in professions that require them to speak in both languages (e.g. librarian, secretary).

\subsection{Materials}

Elicitation materials consist of a set of carrier phrases in French, with 17 target words consisting of $2(5 x), 3(8 x)$ or $4(4 x)$ syl-

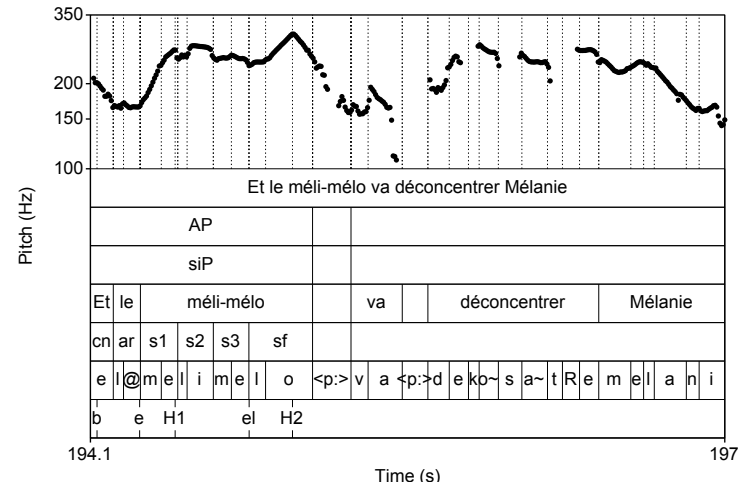

Figure 2: F0 contour of carrier sentence with the token word "méli-mélo" in sentence initial position, followed by a pause (siP). The function words are marked as (cn, for conjunction) and (ar, for article) and syllables are marked according to their position (sf, for final syllable). The full sentence is: "Et le mélimélo va déconcentrer Mélanie" (Engl. And the chaos is going to distract Melanie).

lables. Target words had only sonorant or voiced consonants and were preceded by 1 or 2 monosyllabic function words. The position of the target phrase in the sentence was initial (si) or medial (sm). Materials were identical to those from [11] who invetigated tonal alignment in Standard French. Materials were chosen because they ensured comparable data regarding the position of both rises, and eventual variation in patterns. In contrast to [11], who included 60 phrases in her study, we added two more target words with 3 and 4 syllables, summing a total of 68 carrier phrases.

\subsection{Procedure}

Recordings were made with a Zoom H6 in a quite room. Materials were provided printed on paper and partcipants had enough time to familiarise themselves prior to recording. Sound files were segmented and force aligned in WebMAUS, using a parameter model based on SAMPA [17] and then manually corrected. As exemplified in Figure 2, first, the target APs, position, and pauses were identified. Subsequently, tones and syllable boundaries were marked by hand. Due to misspellings, disfluencies or pitch track errors $15 \%$ of the data had to be discarded. We are reporting results from 292 APs. Data were extracted using the EmuR [18] and R Praat packages [19].

\subsection{Analysis}

The experiment included two positions for the target tokens, sentence initial (si) and sentence medial (sm). Lifou speakers tended to insert pauses between the target NPs and subsequent NPs or VPs. These cases were coded as sentence initial with following pause (siP) and sentence medial with pause (smP). Measurements of fundamental frequency were taken for the tonal target $(\mathrm{Hi})$, the low tone preceding the late rise $(\mathrm{L})$, and the tonal target $\left(\mathrm{H}^{*}\right)$. Additionally, in sentence medial position measurements were taken for the $\mathrm{H}^{*}$ tone of the preceding $\mathrm{AP}(\mathrm{pH})$. To provide a psycholinguistically more accurate analysis we converted the frequency measured in $\mathrm{Hz}$ into semitones. This is in agreement with [20] who demonstrated that the semitones scale best represents intonational equivalence. Figure 3 shows 


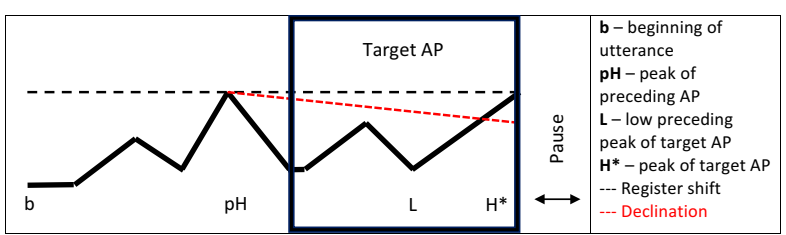

Figure 3: Stylised FO contour of target AP in sentence medial position with pause, and a preceding AP. The black dotted line illustrates the expected register shift at the ip boundary in the Target AP.

the points where measurements were taken in sentence medial position. Values for duration for all syllables in the target APs were also taken. We used the software R [21] and a statistics package [22] to carry out all statistical analysis.

\subsection{Tonal pattern}

Labels for tonal targets were extracted and then associated with the corresponding patterns which were defined according to $[10,11]$. According to [10], for French the initial rise is weaker than the late rise in fundamental frequency and duration. Mean values of $\mathrm{F} 0$ for all $\mathrm{Hi}$ and $\mathrm{H}^{*}$ tones and mean values for the duration of all syllables associated with these tones were measured.

\subsection{Complete reset hypothesis}

To evaluate whether there is a new prosodic level indicated by an insertion of a pause, we compared the $\mathrm{H}^{*}$ tones of sentence medial APs in two conditions ( $\mathrm{sm}$ and smP) and the $\mathrm{H}^{*}$ tones of their preceding APs (labelled as $\mathrm{pH}$, see Figure 3). Recall that in case there is an ip the $\mathrm{H}^{*}$ tone in sentence medial position with pause should show register shift instead of declination. Measurements for $\mathrm{F} 0$ were taken for tonal targets at the peak of $\mathrm{H}^{*}$. Values for $\mathrm{F} 0$ in semitones were fitted into a linear mixed effects model [23] which included 154 observations. A first model included condition (with pause or no pause) and position of rise (target AP or previous AP) as fixed factors, and speaker as random factor. A second model included only condition as fixed factor, and speaker as random factor. We performed a likelihood ratio test using the ANOVA function.

Measurements for the duration of all syllables (see Figure 4) were taken and then fitted into a linear mixed effects model in order to investigate if the position in the carrier phrase and the insertion of the pause had an effect on duration [23]. We employed the step function in order to find the best model and conducted a bonferroni post hoc test to confirm significance of results. The model included 1260 observations and position of the syllable within the Target AP plus position of the AP within the carrier phrase as fixed factors and speaker as random factor.

\subsection{Pitch span hypothesis}

We hypothesised that not only a rise of $\mathrm{F} 0$ causing register shift, but that the use of a greater pitch span could mark the ip boundary. The pitch span value was calculated as the difference in semitones between the $\mathrm{H}^{*}$ and the preceding $\mathrm{L}$ tone. Figure 5 shows the differences of pitch span in semitones at two prosodic levels (AP and ip) and according to the position of the target token within the carrier phrase. Measurements of pitch span were taken and then fitted into a linear mixed effects model in order to asses whether the position in the carrier phrase and the inser-
Table 1: Realization of tonal patterns in Standard French (taken from [11]) and Lifou French.

\begin{tabular}{lll}
\hline Pattern & Standard & Lifou \\
\hline LHiLH* & $50 \%$ & $64 \%$ \\
LH $^{*}$ & $21 \%$ & $20 \%$ \\
LLH* & $18 \%$ & $9 \%$ \\
LHiH* & $4,5 \%$ & $6 \%$ \\
LHi & $4 \%$ & $1 \%$ \\
LH & $2 \%$ & $(1) \%$ \\
\hline
\end{tabular}

Table 2: Comparison of initial $(\mathrm{Hi})$ and late $\left(H^{*}\right)$ rise

\begin{tabular}{llll}
\hline Mean values & in & Hi & $\mathbf{H}^{*}$ \\
\hline F0 & Hz & 222 & 246.7 \\
& st & 13.71 & 13.51 \\
Duration & ms & 191.3 & 302.2 \\
\hline
\end{tabular}

tion of the pause had an effect on it. The model included 244 observations and included position and number of rises as fixed factors and speaker as random factor. We used the step function in order to assess the model and conducted a bonferroni post hoc test to confirm significance of results.

\section{Results}

\subsection{Tonal pattern}

Table 1 shows the proportion of different tonal patterns for the 292 APs annoted in this study. As in [11], with 64\% the canonical /LHiLH*/ pattern represents the most frequent realization in our corpus. Since tones tend to be undershot when the AP has less than 3 syllables the more frequent realization of the canonical pattern is probably caused by the extra tokens with 3 and 4 syllables that were not part of the study of [11] but were included in our corpus. Recall that for French the initial rise is weaker than the late rise in fundamental frequency and duration. Table 2 shows the mean values in hertz and semitones for $(\mathrm{Hi})$ and $\left(\mathrm{H}^{*}\right)$ as well as the mean values in milliseconds for the duration of the syllables containing these tones. As in Standard French in Lifou French the $(\mathrm{Hi})$ and $\left(\mathrm{H}^{*}\right)$ show the same acoustic correlates.

\subsection{Complete reset}

Regarding the register shift at sentence medial position a complete reset to a higher F0 was observed (mean F0 in semitones $\mathrm{pH}=15.20$ vs. mean $\mathrm{H}^{*}$ in $\mathrm{smP}=15.65$ ). These differences were rather small which is why it is not surprising that the likelihood ratio test comparing the two models showed the difference of F0 height is not significant $\left(\chi^{2}(1)=0.1765, p=0.67\right)$. Figure 4 shows the duration in milliseconds of AP final syllables at two prosodic levels (AP and ip) and in the four tested positions (si, $\mathrm{sm}$, siP, smP). It was established that the duration of syllables in position siP is significantly longer than that in si position $\left(\chi^{2}(1)=15.92, \mathrm{p}<0.0001\right)$; also in siP position syllables are significantly longer than at sm position $\left(\chi^{2}(1)=15.92, \mathrm{p}<0.0001\right)$. However, duration is not significantly longer in $\mathrm{smP}$ position when compared to sm position $\left(\chi^{2}(1)=15.92, p=0.18\right)$. 


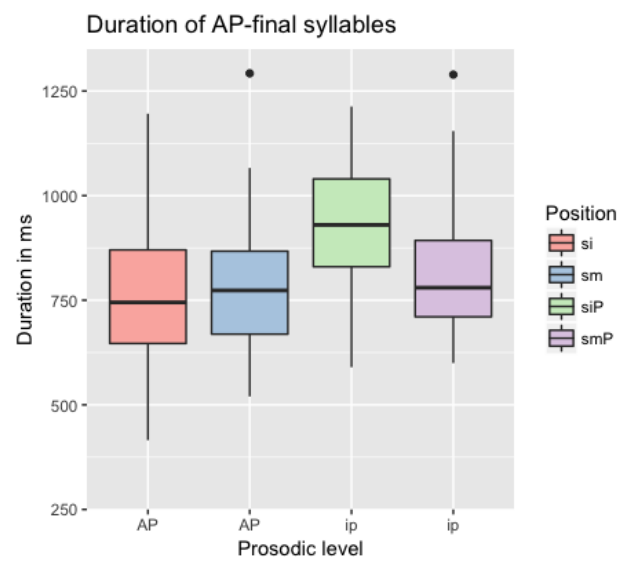

Figure 4: Duration in milliseconds of AP-final syllables measured at two prosodic levels 1. (AP) Accentual Phrase and 2. (ip) intermediate phrase. The legend indicates the position of the target token within the carrier phrase: (si) sentence initial, ( $\mathrm{sm}$ ) sentence medial, (siP) sentence initial with following pause, (smP) sentence medial with following pause.

\subsection{Pitch span}

Figure 5 shows the measurements for pitch span at two prosodic levels (AP and ip) and in the four tested positions (si, sm, siP, $\mathrm{smP}$ ). Recall, pitch span was calculated as the difference in semitones between the $\mathrm{H}^{*}$ and the preceding $\mathrm{L}$ tone at the AP boundary. Pitch span was significantly wider when the AP was followed by a pause ( $\mathrm{siP}$ and smP) thus marking the ip boundary. In sentence initial position pitch span was significantly wider when a break was introduced $\left(\chi^{2}(1)=21.1, \mathrm{p}<0.0001\right)$. In sentence medial position, the effect of pitch span was also significant at the ip-boundary $\left(\chi^{2}(1)=21.1, \mathrm{p}=0.013\right)$.

\section{Discussion and Conclusions}

This preliminary study aimed at describing the realization of the Accentual Phrase in Lifou French by bilingual speakers of Drehu and French. To phonetically investigate the AP we employed an experimental approach based on materials previously used to describe the AP in Standard French [11]. Our goals were twofold, first we wanted to categorize the tonal patterns used when realising the AP and verify the phonetic relization of the tonal targets $\mathrm{Hi}$ and $\mathrm{H}^{*}$. Second, we wanted to investigate the occurrence of an intermediate phrase as a higher level, after the AP in the prosodic hierarchy in French. For this purpose, we investigated register shift, final syllable lengthening and pitch span expansion at the AP boundary.

Results show that the same tonal patterns as in Standard French were used. Similarly, the distribution of the patterns used in Lifou French resembled those of Standard French and the canonical /LHiLH*/ was the most frequently used pattern. Additionally, it was shown that the initial and final peaks (Hi) and $\left(\mathrm{H}^{*}\right)$ phonetically behave the same way as in Standard French with the initial Hi being weaker in fundamental frequency and the corresponding syllable also being shorter when compared to the late $\mathrm{H}^{*}$. Regarding the existence of an intermediate phrase, we first tested the occurrence of register shift and complete reset instead of declination [15]. In our data, we found a global reset of F0 at the ip-break. Although it was found that the fundamental frequency of the late rise in the target $\mathrm{AP}$

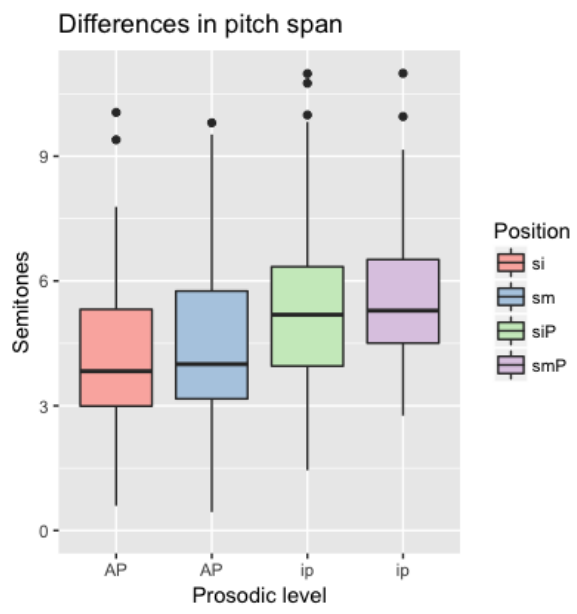

Figure 5: Differences of pitch span measured at two prosodic levels 1. (AP) Accentual Phrase and 2. (ip) intermediate phrase. The legend indicates the position of the target token within the carrier phrase: (si) sentence initial, (sm) sentence medial, (siP) sentence initial with following pause, (smP) sentence medial with following pause.

increased to a higher level than that of the preceding AP, the difference was only small. A statistical analysis confirms differences are not significant. We also investigated final syllable lengthening. In this case, it could be established that only if the break was inserted in sentence initial position the duration of the syllables was significantly longer. Second, we investigated the use of pitch span expansion at the AP boundary. It was found that the expansion resulting from a lowering of the $\mathrm{L}$ and a raising of the $\mathrm{H}^{*}$ tone targets was significantly wider when preceding a pause. The strong pitch span expansion at the right AP boundary confirms the insertion of the intermediate phrase as a further prosodic level. Previous studies on contact varieties of French spoken by bilinguals also speaking languages with word prosodic systems (Sango and Swiss German) [6, 7] suggest the speech flow in French would be segmented in smaller chunks resembling word prosodic systems. Contrary to these studies, we did not find that the speech flow was segmented differently from Standard French but rather that the tonal patterns used are the same. Considering the insertion of an intermediate phrase, we found that complete reset of F0 and final syllable lengthening occur. However, statistical analyses reveal that these are not the strongest marker of the ip-break. Instead, it was found that the use of pitch span expansion was significantly wider at the ip boundary than at the AP boundary. In light of this result, we consider pitch span to be the most salient phonetic cue marking the intermediate phrase in Lifou French. Future research will look in more detail at the realization of tonal alignment and segmental anchoring in Lifou French. Similarly, it is our aim to investigate the phonetic implementation of accentual prominence and phrasing in Drehu, the other language of the bilinguals.

\section{Acknowledgements}

Special thank to the participants who made this study possible. Also thanks to the anonymous reviewers and their helpful comments. This research was conducted with support from the ARC Centre of Excellence for the Dynamics of Language (Project ID: CE140100041). 


\section{References}

[1] "Recensement général de la population," 2009.

[2] J. Vernaudon, "Linguistic Ideologies: Teaching Oceanic Languages in French Polynesia and New Caledonia," The Contemporary Pacific, vol. 27, no. 2, pp. 433-462, 2015.

[3] S.-A. Jun and C. Fougeron, "Realizations of accentual phrase in French intonation," Probus, vol. 14, no. 1, pp. 147-172, 2002.

[4] M.-H. Lenormand, "La phonologie du mot en lifou (îles loyalty)," Journal de la Société des Océanistes, vol. 10, no. 10, pp. 91-109, 1954

[5] D. T. Tryon, Dehu grammar. Australian National University, 1968.

[6] M. Avanzi, P. Dubosson, S. Schwab, and N. Obin, "Accentual Transfer from Swiss-German to French a Study of Français Fédéral," in Interspeech, 2012, pp. 1-1.

[7] G. Bordal, M. Avanzi, N. Obin, and A. Bardiaux, "Variations in the realization of the french accentual phrase in the light of language contact," in Speech Prosody, 2012, pp. 1-1.

[8] P. Welby, "French intonational structure: Evidence from tonal alignment," Journal of Phonetics, vol. 34, no. 3, pp. 343-371, 2006.

[9] V. Pasdeloup, "A prosodic model for french text-to-speech synthesis: A psycholinguistic approach," Talking Machines. Theories Models and Designs, pp. 335-348, 1992.

[10] S.-A. Jun and C. Fougeron, "A phonological model of french intonation," Intonation: Analysis, modeling and technology, pp. 209 242, 2000.

[11] P. S. Welby, "The slaying of lady Mondegreen, being a study of French tonal association and alignment and their role in speech segmentation," Ph.D. dissertation, The Ohio State University, 2003.

[12] B. M. B. Post, Tonal and phrasal structures in French intonation Thesus The Hague, 2000, vol. 34

[13] M. D'Imperio and A. Michelas, "Embedded register levels and prosodic phrasing in french," in Speech Prosody, 2010, p. 4.

[14] A. Michelas and M. D'Imperio, "When syntax meets prosody: Tonal and duration variability in french accentual phrases," Journal of Phonetics, vol. 40, no. 6, pp. 816-829, 2012.

[15] M. D'Imperio and A. Michelas, "Pitch scaling and the interna structuring of the intonation phrase in french," Phonology, vol. 31 no. 1, pp. 95-122, 2014.

[16] L. M. Gertken, M. Amengual, and D. Birdsong, "Assessing language dominance with the bilingual language profile," Measuring L2 proficiency: Perspectives from SLA, pp. 208-225, 2014.

[17] T. Kisler, U. Reichel, and F. Schiel, "Multilingual processing of speech via web services," Computer Speech \& Language, vol. 45 pp. 326-347, 2017.

[18] R. Winkelmann, K. Jaensch, S. Cassidy, and J. Harrington, emuR Main Package of the EMU Speech Database Management System, 2017, $\mathrm{r}$ package version 0.2.3.

[19] T. Bořil and R. Skarnitzl, "Tools rpraat and mpraat," in International Conference on Text, Speech, and Dialogue. Springer, 2016, pp. 367-374.

[20] F. Nolan, "Intonational equivalence: an experimental evaluation of pitch scales," in Proceedings of the 15th International Congress of Phonetic Sciences, Barcelona, vol. 39, 2003.

[21] R Core Team, R: A Language and Environment for Statistical Computing, R Foundation for Statistical Computing, Vienna, Austria, 2017. [Online]. Available: https://www.R-project.org/

[22] D. Bates, M. Mächler, B. Bolker, and S. Walker, "Fitting linear mixed-effects models using lme4," Journal of Statistical Software, vol. 67, no. 1, pp. 1-48, 2015.

[23] B. Winter, "Linear models and linear mixed effects models in $\mathrm{r}$ with linguistic applications," arXiv preprint arXiv:1308.5499, 2013. 